\title{
Influence of electron-acoustic phonon scattering on off-resonant cavity feeding within a strongly coupled quantum-dot cavity system
}

\author{
S. Hughe\& and P. Yao \\ Department of Physics, Queen's University \\ Kingston, ON KYL $3 N 6$ Canada \\ F. Milde and A. Knorr \\ Institut für Theoretische Physik, Nichtlineare Optik und Quantenelektronik, \\ Technische Universität Berlin, Hardenbergstraße 36, PN 7-1 10623 Berlin, Germany
}

\begin{abstract}
D. Dalacu, K. Mnaymneh, V. Sazonova, P. J. Poole, G. C. Aers, J. Lapointe, R. Cheriton, and R. L. Williams Institute for Microstructural Sciences, National Research Council, Ottawa, Canada K1A OR6
\end{abstract}

\begin{abstract}
We present a medium-dependent quantum optics approach to describe the influence of electronacoustic phonon coupling on the emission spectra of a strongly coupled quantum-dot cavity system. Using a canonical Hamiltonian for light quantization and a photon Green function formalism, phonons are included to all orders through the dot polarizability function obtained within the independent Boson model. We derive simple user-friendly analytical expressions for the linear quantum light spectrum, including the influence from both exciton and cavity-emission decay channels. In the regime of semiconductor cavity-QED, we study cavity emission for various exciton-cavity detunings and demonstrate rich spectral asymmetries as well as cavity-mode suppression and enhancement effects. Our technique is nonperturbative, and non-Markovian, and can be applied to study photon emission from a wide range of semiconductor quantum dot structures, including waveguides and coupled cavity arrays. We compare our theory directly to recent and apparently puzzling experimental data for a single site-controlled quantum dot in a photonic crystal cavity and show good agreement as a function of cavity-dot detuning and as a function of temperature.
\end{abstract}

PACS numbers: 42.50.Ct, 78.67.Hc, 42.50.Pq

\section{INTRODUCTION}

The influence of electron-acoustic phonon scattering is a well known effect in semiconductor quantum dot (QDs). Due to the interplay of phonon emission and absorption, the longitudinal acoustic (LA) phonon bath manifests in spectral lineshapes that are highly asymmetric at low temperature, and sit on the background of the symmetric zero phonon line (ZPL). The characteristic spectral lineshape of LA phonon scattering on electron-hole pairs ("excitons") in quasi-homogeneous semiconductor structures is well described through the independent Boson model (IBM) $)^{1-4}$, and IBM simulations have shown good agreement with experiments. In addition to the IBM lineshape, the broadening of the ZPL is usually described phenomenologically, though this can be reliably fit to experiments ${ }^{5}$; however, there is still some controversy as to the origin of the ZPL broadening which can include contributions from radiative broadening, spectral diffusion, anharmonicity effects $\frac{6.7}{.7}$, phonon scattering from interfaces ${ }^{8,9}$, and a modified phonon spectrum 10 . While it is well known that the phonons cause the exciton lineshapes to be highly non-Lorentzian (stemming from nonMarkovian decay), most of the present QD cavity-QED theories only add in a Lorentzian broadening mechanism for the QD excitons, e.g., at the level of a Markovian master equation.

Recently, there have been several studies of the role of electron-phonon coupling in semicondutor QD cav- ity systems. Wilson-Rae and Imamoglư 11 treated the phonon interaction with QDs using polaron Green functions and derived an analytic absorption lineshape when the dot and cavity are on resonance; in the polaron representation, new phonon-induced interaction terms are introduced exactly, while a second-order Born approximation was applied to include "residual" exciton-photonphonon coupling effects. Polaron approaches have also been recently employed to successfully describe phononinduced decay of optical pulse-excited QDs in the absence of any cavity coupling 12 . Milde et al $\underline{13}$ numerically solved the IBM and coupled the QD susceptibility to a photonic crystal cavity system through a semiclassical Green function approach, demonstrating asymmetries in the on-resonance Rabi doublet and the effect of increasing temperature for both the cavity emitted spectra and the side-coupled waveguide transmission. Xue et al $\stackrel{14}{=}$ have applied perturbation theory to study the phonon-induced decoherence on vacuum Rabi oscillations as a function of detuning between the cavity mode and exciton. Kaer et $a l \stackrel{15}{\underline{1}}$ also explored off-resonant interactions between the QD and a cavity using a numerical solution to the system master equations within a time-convolutionless approach. Ota et al $\stackrel{16}{\underline{16}}$ numerically solved the Wilson-Rae and Imamoglŭ system master equation 11 , and demonstrated the importance of asymmetric off-resonance coupling and non-Markovian relaxation, finding good agreement with their experiments. Hohenester ${ }^{17}$ introduced a useful model to derive an effective phonon-mediated scat- 
tering rate (see also Ref $\stackrel{14}{ }$ ), and has found good agreement with experiments in the weak coupling regime $\underline{18}$.

From an experimental viewpoint, significant offresonant coupling between an exciton and a cavity has been seen in a number of semiconductor QD-cavity systems, e.g., see Refs $\underline{\underline{19}-23}$; and several theoretical and experimental works have tried to explain the basic coupling mechanisms $24-28$, most of which stem from the exciton broadening mechanisms and the simple physics of two coupled oscillators. Given the importance of exciton decay processes on the QD-cavity coupling, one can therefore expect that the interaction of phonons can play a qualitatively important role when certain coupling conditions are met. However, while the aforementioned theoretical phonon studies are interesting in their own right, unfortunately none of them present simple analytical spectra that allow one to explore a wide range of coupling phenomena both on- and off-resonance with a strongly coupled cavity. Thus many of the experimental - and theoretical - groups continue to use the only available analytical formulas with no phonon coupling directly included, e.g., see Ref. 29 .

Here we apply a photon Green function method to derive useful analytical spectra, with phonons included to all orders through the exciton polarizability. Although a similar semi-classical Green function approach was presented by Milde et al $\stackrel{13}{\underline{13}}$, only on-resonance conditions were studied, and the IBM was solved numerically. Tarel and Savona ${ }^{30}$ have recently presented a semi-classical Green function spectra with phonons included to second order (also previously presented in Ref $\underline{13}$ ), where numerical solutions of the phonon baths were exploited. We first study leaky cavity emission with and without phonons and connect the results to the recent work of Ota et $a l .16$, and find good qualitative agreement with their observations, namely pronounced asymmetries for high energy or low energy cavity coupling and an asymmetric vacuum Rabi doublet (at low temperatures, $T \approx 4 \mathrm{~K}$ ); we also demonstrate that phonon-induced cavity suppression can occur, which is an effect that stems from the real part of the phonon self-energy. Secondly, we compare directly with recent experimental measurements by Dalacu et $a l \stackrel{29}{\underline{29}}$, who studied QD-cavity coupling in single sitecontrolled QDs and found that the data could not be fit without adding in some unknown (detuning-dependent) cavity pump term; in contrast, we show that this data can be well reproduced using our analytic model with no cavity pump term included at all. Importantly, our spectra can be applied to a wide range of systems, including waveguides, and it rigorously applies to both weak and strong coupling regimes, and contains phonons to all orders (at the level of the IBM). We also show higher temperature results and systematically compare these with experimental data. In our experimental-theory analysis, we include two decay channels, accounting for both the radiation-mode emission and the leaky cavity emission.

Our paper is organized as follows. In Sec. III, we introduce the basic theory and analytical formulas for calcu- lating the emitted spectra from a strongly coupled QDcavity system in the presence of electron-acoustic phonon coupling. Example computed emission spectra with and without phonon coupling are shown in Sec. III] Section IV compares our theory with recent data on site controlled single QDs in photonic crystal cavities 29 , and demonstrates the significant influence of electron-phonon coupling. In Sec. D] we conclude.

\section{THEORY AND ANALYTICAL SPECTRA}

For this work, we are interested in the linear spectrum and thus consider a QD-cavity system that is weakly pumped, incoherently, where the emission dynamics stem from an excited electron-hole pair. In general, there have been several theoretical approaches to this problem in the literature. Two of the most powerful methods include Green function approaches 28 and quantum master equation techniques $31-33$. A major advantage of the Green function approach is that one can obtain analytical spectra for any inhomogeneous and lossy structures, including lossy metamaterial waveguides 34 and a variety of coupled cavity-waveguide systems ${ }^{35,36}$. In contrast, a key advantage of the master equation approach is the ease with which it adds in additional dissipation effects such as pure dephasing, though at the level of obtaining emission spectra, both Green function and master equation approaches can be equivalent. A significant disadvantage of the master equation approach is that it is typically limited to simple leaky cavity systems with a Lorentzian decay, i.e., Markovian theory. Master equation solutions can also consider more realistic initial conditions, such as those obtained through steady-state pumping.

The general Green function approach to obtaining the electric-field operator has been described elsewhere $28,37,38$. Here we will briefly highlight the theoretical background and concentrate on presenting the general expressions for the field operator and the spectra. At first we neglect non-radiative broadening on the exciton decay, which allows us to obtain the exact analytical field operator without coupling to phonons. Specifically, we use a canonical Hamiltonian that quantizes the macroscopic electromagnetic fields, and exploit the dipole approximation for the QD-medium coupling:

$$
\begin{aligned}
\hat{H} & =\hbar \omega_{x} \hat{\sigma}_{x}^{+} \hat{\sigma}_{x}^{-}+\sum_{\lambda} \hbar \omega_{\lambda} \hat{a}_{\lambda}^{\dagger} \hat{a}_{\lambda} \\
& -i \hbar \sum_{\lambda}\left(\hat{\sigma}_{x}^{-}+\hat{\sigma}_{x}^{+}\right)\left(g_{\lambda} \hat{a}_{\lambda}-g_{\lambda}^{*} \hat{a}_{\lambda}^{\dagger}\right),
\end{aligned}
$$

where $\hat{a}_{\lambda}$ represents the field mode operators and $\hat{\sigma}_{x}^{+/-}$ are the Pauli operators of the QD excitons, $\omega_{\lambda}$ is the eigenfrequency corresponding to the transverse modes of the system $\left[\mathbf{f}_{\lambda}(\mathbf{r})\right]$, excluding the dot; $g_{\lambda}$ is the field-dot coupling coefficient, defined through $g_{\lambda}=\sqrt{\frac{\omega_{\lambda}}{2 \hbar \varepsilon_{0}}} \boldsymbol{\mu}_{x}$. $\mathbf{f}_{\lambda}(\mathbf{r})$, with $\boldsymbol{\mu}_{x}=\boldsymbol{n}_{x} \mu_{x}$ the optical dipole moment of 
the exciton, aligned along $\boldsymbol{n}_{x}$ (a unit vector). We consider only one target exciton in the spectral region of interest for the coupled QD and assume that coupling to the other polarized exciton is negligible. The Heisenberg equations of motion for the operators can be used to derive the electric field operator ${ }^{28.38}$. Considering a weak excitation condition (i.e., we neglect higher-order photon-correlation effects, which is valid in these systems for weak powers 33 ), and assuming an excited QD in vacuum, we derive the quantum field operator ${ }^{28}$ :

$$
\hat{\mathbf{E}}(\mathbf{r}, \omega)=\frac{\mathbf{G}\left(\mathbf{r}, \mathbf{r}_{d} ; \omega\right) \cdot \hat{\mathbf{d}}_{x}(\omega) / \varepsilon_{0}}{1-\mathbf{n}_{x} \cdot \mathbf{G}\left(\mathbf{r}_{d}, \mathbf{r}_{d} ; \omega\right) \cdot \mathbf{n}_{x} \alpha_{x}(\omega)},
$$

where $\mathbf{r}_{d}$ is the QD position, $\alpha_{x}(\omega)=\frac{\mu_{x}^{2}}{\hbar \varepsilon_{0}} \frac{2 \omega_{x}}{\omega_{x}^{2}-\omega^{2}}$ is the bare (no radiative or non-radiative coupling) exciton polarizability, and $\hat{\mathbf{d}}_{x}(\omega)=-i \boldsymbol{\mu}_{x}\left[\hat{\sigma}_{x}^{-}(t=0) /\left(\omega-\omega_{x}\right)+\right.$ $\left.\hat{\sigma}_{x}^{+}(t=0) /\left(\omega+\omega_{x}\right)\right]$ is a quantum dipole source that originates from the excited QD. Without phonon interactions, the above operator is exact within the stated model approximations. The propagator, $\mathbf{G}\left(\mathbf{r}, \mathbf{r}^{\prime} ; \omega\right)$, is the transverse ${ }^{33}$ photon Green function of the medium, without any $Q D$, and for cavity systems it can be easily written down analytically in terms of the cavity mode decay (cavity emission) and the radiation-mode decay (dot emission) ${ }^{28}$. Although electric field pumping (e.g., cavity pumping) can be included in a straightforward way, we are primarily interested in the spectra from an excited (and single) QD, so we treat the initial field as in vacuum.

With a knowledge of the medium-dependent Green functions, and a suitable initial condition for exciting the material system, one can conveniently obtain the analytic emitted spectrum, in any general structure, from ${ }^{28}$ $S(\mathbf{r}, \omega)=\left\langle[\hat{\mathbf{E}}(\mathbf{r}, \omega)]^{\dagger} \hat{\mathbf{E}}(\mathbf{r}, \omega)\right\rangle$. The above expressions are exact, and no assumption has been made about the form of the medium.

For a semiconductor cavity system such as a planar photonic crystal cavity, one easily obtains the cavity emitted spectrum and the radiation-mode emitted spectrum $^{28}$, analytically,

$$
\begin{aligned}
& S_{r}(\mathbf{r}, \omega)=F_{r}(\mathbf{r}) \Gamma_{\mathrm{rad}} \times \\
& \left|\frac{\omega_{x}+\omega}{\omega_{x}^{2}-\omega^{2}-i \omega \Gamma_{x}-\frac{4 g^{2} \omega_{x} \omega_{c}}{\omega_{c}^{2}-\omega^{2}-i \omega \Gamma_{c}}}\right|^{2}, \\
& S_{c}(\mathbf{r}, \omega)=F_{c}(\mathbf{r}) \Gamma_{c} \times \\
& \left|\frac{\frac{2 g \omega_{c}\left(\omega_{x}+\omega\right)}{\omega_{c}^{2}-\omega^{2}-i \omega \Gamma_{c}}}{\omega_{x}^{2}-\omega^{2}-i \omega \Gamma_{x}-\frac{4 g^{2} \omega_{x} \omega_{c}}{\omega_{c}^{2}-\omega^{2}-i \omega \Gamma_{c}}}\right|^{2},
\end{aligned}
$$

with the total spectrum $S_{t}(\mathbf{r}, \omega)=S_{r}(\mathbf{r}, \omega)+S_{c}(\mathbf{r}, \omega)$. We emphasize that radiative coupling to the cavity system is fully included by coupling to both the continuum of radiation modes and the leaky cavity modewith a decay rate given by $\Gamma_{c}$. Additional broadening of the ZPL has also been included, phenomenologically, through $\Gamma_{x} \equiv \Gamma_{\text {rad }}+\Gamma^{\prime}$, with $\Gamma^{\prime}$ due to pure dephasing processes; in general, for $\mathrm{cw}$ (continuous wave) pumping, both phonon effects and spectral diffusion will enhance $\Gamma^{\prime}$, and $\Gamma^{\prime}$ is also known to be temperature dependent ${ }^{\underline{5}}$. It is important to note that in a planar photonic crystal structure, both $S_{r}$ and $S_{c}$ photon decay channels contribute to vertical photon emission. The radiation-mode decay is due to coupling to the continuum of radiation modes above the slab light line ${ }^{28}$; and the $F_{c / r}(\mathbf{r})$ represent the geometrical factors which will depend upon the collection geometry of light emission. For a micropillar cavity system ${ }^{39}$, typically only the cavity emission is required in an identical form to above, and so the prescriptions apply to a wide range of semiconductor cavity systems.

To include phonon interactions in a simple but rigorous way, we assume that the cavity and phonon correlation functions can be decoupled, and add in the phonon polarizability via the known phonon self-energy, $\Sigma_{p h}(\omega)$, from the IBM In essence, we are then considering the optical polarizability of the QD in the presence of phonons, as the exact perturbation to the medium. Consequently, one has a slight modification to the above spectra,

$$
\begin{aligned}
& S_{r}(\mathbf{r}, \omega)=F_{r}(\mathbf{r}) \Gamma_{\mathrm{rad}} \times \\
& \left|\frac{\omega_{x}+\omega}{\omega_{x}^{2}-\omega^{2}-i \omega \Gamma_{x}-\omega \Sigma_{p h}(\omega)-\frac{4 g^{2} \omega_{x} \omega_{c}}{\omega_{c}^{2}-\omega^{2}-i \omega \Gamma_{c}}}\right|^{2}, \\
& S_{c}(\mathbf{r}, \omega)=F_{c}(\mathbf{r}) \Gamma_{c} \times \\
& \left|\frac{\frac{2 g \omega_{c}\left(\omega_{x}+\omega\right)}{\omega_{c}^{2}-\omega^{2}-i \omega \Gamma_{c}}}{\omega_{x}^{2}-\omega^{2}-i \omega \Gamma_{x}-\omega \Sigma_{p h}(\omega)-\frac{4 g^{2} \omega_{x} \omega_{c}}{\omega_{c}^{2}-\omega^{2}-i \omega \Gamma_{c}}}\right|^{2},
\end{aligned}
$$

where the cavity emission is similar in form to the one presented by Tarel and Savona ${ }^{30}$, where phonons were included to second-order and a rotating-wave approximation was made. As limits, we obtain the correct IBM spectral form for exciton decay, and earlier derived spectra for semiconductor cavities 28 . We also obtain ZPL broadening associated with the leaky cavity system.

In the spirit of deriving a simple analytic solution, with phonons included to all orders, the strategy is to use an analytic phonon self-energy at the level of the IBM. To do this, we exploit phonon spectral functions, similar to the ones used by Wilson-Rae and Imamoglư 11 , but we use a more appropriate spectral function for phonon interactions via a deformation potential $\stackrel{40}{ }$; similar spectral function are commonly used when describing LA-phonon coupling. To obtain the phonon self-energy, the IBM time-dependent phase must be added into the Lorentzian decay model for the exciton; this is obtained from

$$
\psi(t)=\int_{0}^{\infty} d \omega J(\omega) / \omega^{2}[\operatorname{coth}(\beta \hbar \omega / 2) \cos (\omega t)-i \sin (\omega t)],
$$

that describes the electron-LA-phonon interaction, at temperature $T=1 / \beta k_{b}$; we consider electron-phonon interactions via a deformation potential coupling which is 
known to account for the major phonon interactions in our considered QD. Although one may have LO interactions as well, these give no dephasing. We considering a spherical QD model, with similar electron localization lengths in the valence and conduction bands $\left(l_{e}=l_{h} \approx 5 \mathrm{~nm}\right)$. Generalizing to include electrons and holes with different localization lengths is straightforward, but the equations become more cumbersome. Thus, spectral function can be conveniently defined as 40

$$
J(\omega)=a_{p} \omega^{3} \exp \left(-\omega^{2} / 2 \omega_{b}^{2}\right),
$$

where we use $\omega_{b}=1 \mathrm{meV}$ and $a_{p} /(2 \pi)^{2}=0.06 \mathrm{ps}^{2}$ as representative numbers for InAs type QDs ${ }^{14,41}$. The deformation coupling constant used here is somewhat smaller

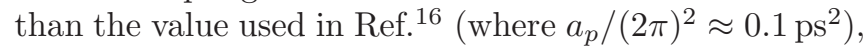
though in the literature there is no well accepted values for InAs QDs; moreover, the dimensionless Huang-Rhys, $S_{H R}=a_{p} /(2 \pi)^{2} c_{l}^{2} / l_{e / h}^{2} \approx 0.034\left(\right.$ where $c_{l}=3800 \mathrm{~m} / \mathrm{s}$ is the speed of sound), has been shown to be significantly enhanced in QDs (from its bulk value); for example, for InAs/GaAs QDs, $S_{H R}=0.01-0.5\left(\operatorname{Ref}^{42}\right)$ and $S_{H R}=0.5$ (Ref $\left.{ }^{43}\right)$ have been reported, and various mechanisms for such enhancements have been proposed, including defects and non-adiabatic effects. The phonon interactions also result in a polaron shift, $\Delta=$ $\int_{0}^{\infty} d \omega J(\omega) / \omega=S_{H R} \omega_{b} \sqrt{\pi / 2} \approx 42 \mu \mathrm{eV}$. In what follows below, we neglect the polaron shift as it merely adds a fixed frequency, and we can always redefine $\omega_{x}$, so our $\omega_{x}$ includes the polaron shift. We also point out that there will likely be other non-diagonal phonon coupling as well and so in principle the phonon parameters above could be varied and used to fit experiments.

With the above analytical form for the phonon bath, the time-dependent polarizability takes the form $\alpha_{x}(t)=$ $\alpha_{x}(0) \exp \left[-i\left(w_{x}+\Delta\right) t+\psi(t)\right]$, and the frequency dependent polarizability is obtained from a simple Fourier transform, yielding

$$
\alpha_{x}(\omega)=\frac{d^{2} 2 \omega_{x} / \hbar \varepsilon_{0}}{\omega_{x}^{2}-\omega^{2}-i \omega \Gamma_{x}-\omega \Sigma_{p h}(\omega)} .
$$

To help better explain the phonon coupling effects shown later, in Fig. 11 we show the phonon self-energies for the two different temperatures of $T=4 \mathrm{~K}$ (a) and $T=40 \mathrm{~K}$ (b). We recognize a local minimum in $\operatorname{Im}\left[\Sigma_{p h}\right]$ (red-solid) near $\omega_{x}$, a maximum in $\operatorname{Im}\left[\Sigma_{p h}\right]$ near $1 \mathrm{meV}$, and significant $\operatorname{Re}\left[\Sigma_{p h}\right]$ (blue-dashed) over a broad spectral range. These results imply that both the real and imaginary contributions will have a significant impact on phonon coupling effects in the regime of cavity-QED.

As mentioned above, our general methodology is similar in spirit to a semi-classical approach. Specifically, we have assumed a well defined spectral lineshape for the QD susceptibility, and then coupled this QD frequency response - with phonons included selfconsistently - to the medium-dependent Green functions to obtain the analytical spectra. Comparing with the approach of Wilson-Rae and Imamoglü, they have in their system Hamiltonian a phonon-modified cavity coupling rate ${ }^{11} g \rightarrow g\langle B\rangle$, with $\langle B\rangle=$ $\exp \left(-0.5 \int_{0}^{\infty} d \omega J(\omega) \operatorname{coth}(\beta \hbar \omega / 2) / \omega^{2}\right)$, which, using the parameters above for $T=4-40 \mathrm{~K}$, is around $\langle B\rangle=$ $0.91-0.55$. We do not have this term explicitly, however our self-energy term naturally includes this coupling, and to all orders. To make this clearer, we can rewrite the solution as, e.g., for the cavity-mode emission,

$$
\begin{gathered}
S_{c}(\mathbf{r}, \omega)=F_{c}(\mathbf{r}) \Gamma_{c} \times \\
\left|\frac{\frac{2 g \omega_{c}\left(\omega_{x}+\omega\right)}{\omega_{x}^{2}-\omega^{2}-i \omega \Gamma_{x}}\left(1+\frac{\omega \Sigma_{p h}}{\omega_{x}^{2}-\omega^{2}-i \omega \Gamma_{x}}+\cdots\right)}{\omega_{c}^{2}-\omega^{2}-i \omega \Gamma_{c}-\frac{4 g^{2} \omega_{x} \omega_{c}}{\omega_{x}^{2}-\omega^{2}-i \omega \Gamma_{x}}\left(1+\frac{\omega \Sigma_{p h}}{\omega_{x}^{2}-\omega^{2}-i \omega \Gamma_{x}}+\cdots\right)}\right|^{2},
\end{gathered}
$$

where the real part of the phonon self-energy causes a reduction in the $g$ coupling. Such an effect was previously pointed out before for on-resonance coupling between a cavity and an exciton ${ }^{13}$.

\section{THEORETICAL SIMULATIONS AND PREDICTIONS}

We first clarify that with no phonon coupling included, then our Green function technique yields identical normalized spectra to a master equation approach in the low power limit. Specifically, we use the equations in Ref $^{33}$, with a low-power incoherent exciton pump, and we compute the total spectra from both cavity emission and QD emission in the presence of pure dephasing; with the Green function approach, we define $\Gamma_{Z P L}=\Gamma_{\mathrm{rad}}+\Gamma^{\prime}$. These spectral forms are found to be identical, as previously discussed $^{28}$, so the general phenomenon of cavity feeding is not unique to pure dephasing processes. The
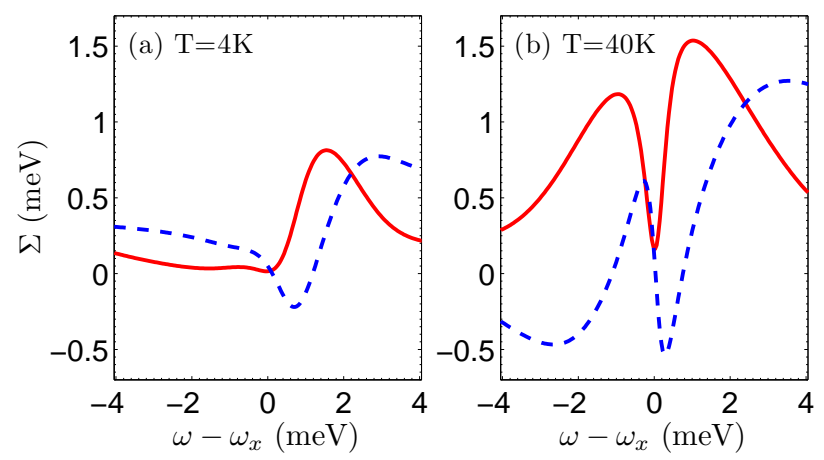

FIG. 1: (Color online) Phonon self energies for InAs QDs, for two different temperatures $(T=4,40 \mathrm{~K})$, where the red-dark curves represent the imaginary contribution and the grey-light curves represent the real contribution. The broadening parameters are $\Gamma_{\mathrm{rad}}=2 \mu \mathrm{eV}$ and $\Gamma_{x}^{\prime}=75 \mu \mathrm{eV}$. As discussed in the text, $\omega_{x}$ is considered to already include a polaron shift. 

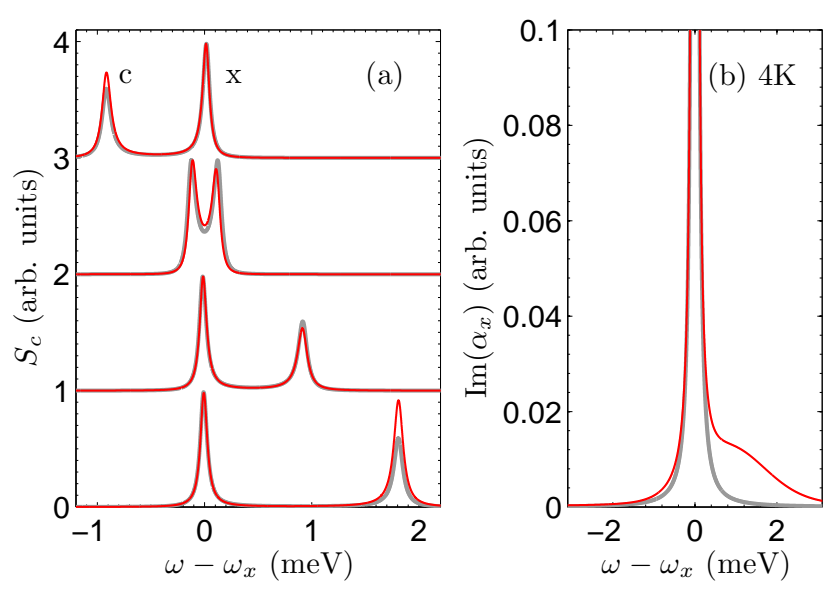

FIG. 2: (Color online) (a) Cavity-emitted spectra for various QD-cavity detunings, obtained for a temperature of $T=4 \mathrm{~K}$. The red-dark and grey-light curves display the Green function solution with and without LA-phonon interactions. The corresponding imaginary part of the polarizability with (reddark curve) and without (grey-light curve) phonon coupling is shown in (b). The lower frequency phonon bath is suppressed in part due to the low temperatures and in part due to the relatively large $\mathrm{ZPL}$. The polaron shift, $\Delta \sim 30 \mu \mathrm{eV}$, is not included as this just adds in a fixed resonance shift for all detunings and temperatures. The parameters are $\Gamma_{\mathrm{rad}}=2 \mu \mathrm{eV}, \omega_{x}=830 \mathrm{meV}, \Gamma_{x}^{\prime}=75 \mu \mathrm{eV}, \Gamma_{c}=100 \mu \mathrm{eV}$, and $g=0.13 \mathrm{meV}$ (see Ref. ${ }^{46}$ ).

only difference is the overall magnitude, which is due to the different initial conditions, though these can be made equivalent as well. Any exciton broadening (radiative or non-radiative) will feed the cavity mode in the cavitymode emission, and scale with $g^{2}$. However, the distinction of pure dephasing plays a much more important role for computing higher-order quantum correlations 45 .

In Fig. 2(b), we show the imaginary part of the QD polarizability (which is proportional to the absorption) with and without coupling to phonons via the IBM; this calculation was obtained at a temperature of $4 \mathrm{~K}$, and we clearly obtain the familiar spectral form of the IBM

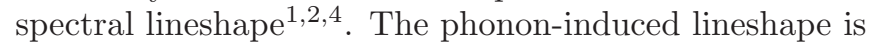
subsequently used to obtain the modified spectra with phonon coupling. In Fig. 2(a) we plot the Green function cavity spectra solution with (red-dark curve) and without (grey-light curve) phonons, where the QD and cavity parameters are given in the figure caption. At zero detuning, we recover an asymmetric Rabi splitting in agreement with Refs $\frac{13,16}{2}$; in addition, for small detunings, a positive detuning gives a cavity suppression, while a negative detuning gives a cavity enhancement. These are due to the significant frequency shifts from the real part of the phonon self-energy, whose effects would be absent in an effective rate approximation. This suppression, followed by an enhancement, would exacerbate the effects of cavity coupling for positive detunings, and depend upon the spectral form of the phonon bath. The off-resonant
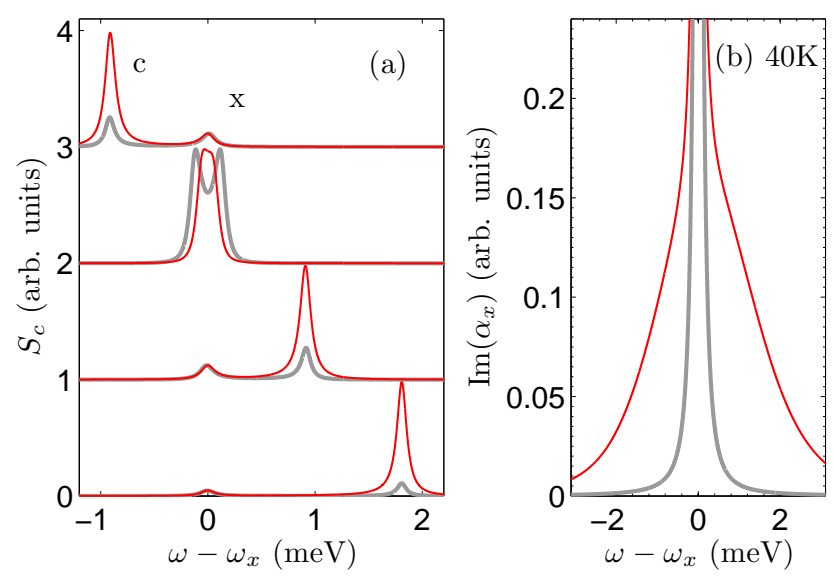

FIG. 3: (Color online) As in Fig. 2 but with $T=40 \mathrm{~K}$ and $\Gamma^{\prime}=150 \mu \mathrm{eV}$.

cavity peak primarily stems from $S_{c}$ and scales with $g^{2}$.

It is also worth stressing that the asymmetric vacuum Rabi doublet is an effect that cannot be predicted with a standard master equation approach, nor by using an effective cavity feeding rate $\underline{18}$. As pointed out some time ago by Carmichael and Walls $\frac{47}{7}$, the usual decomposition of the system Hamiltonian to include only the noninteracting QD and cavity parts does not satisfy the detailed balance condition 47 . This is due to the fact that the cavity and the QD systems are internally coupled, and modified master equation must be derived to account for this internal coupling. In fact, the polaron transformation used by Wilson-Rae and Imamoglự 11 adopts such an approach, and so their transformed system Hamiltonian leads to the correct form of the density operator - since it preserves detailed balance conditions ${ }^{47}$. Physically the asymmetric vacuum Rabi splitting $\frac{13}{}$, which has recently been measured $\frac{16}{6}$, comes form the fact that the two coupled Rabi peaks sample different parts of the asymmetric phonon bath.

Since we include phonons to all orders, it is also possible to study elevated temperatures, which is highly desired from a device viewpoint. In Fig. 3 we repeat the same calculation as before, but at $T=40 \mathrm{~K}$ (chosen primarily to connect to the experiments below), and the ZPL has been broadened (doubled) to be consistent with experiment $t^{\underline{5}}$. Again, we see the same phonon coupling trends as before, but now with a significantly larger phonon feeding to the cavity mode. Also, the effect of the cavity coupling parameters is significantly reduced by about $50 \%$, resulting in a vanishing Rabi splitting on resonance when phonons are included. All of these predicted trends are consistent with the recent experiments of Ota et al $\stackrel{16}{\underline{n}}$. 


\section{COMPARISON WITH RECENT EXPERIMENTAL DATA ON A SINGLE QD- PHOTONIC CRYSTAL CAVITY SYSTEM}

We now apply our theory to help explain the recent model discrepancies that were employed for simulating experimental data. Earlier this year, Dalacu et al $\underline{29}^{29}$ reported measurements on a single QD-cavity system at low power and, using a master equation theory of Refs $\stackrel{33,48}{\underline{4}}$, and clearly demonstrated that an extra cavity pump term had to be included by hand to explain their data. The cavity pump contribution was found to be cavity-QD detuning-dependent. The origin of this cavity pump was unknown and cited to be somewhat mysterious for their excitation conditions and sample, but similar couplings have been shown already to be due to electron-phonon coupling ${ }^{14,16,18}$. The site-controlled QD allows one to suppress other extraneous cavity feeding mechanisms ${ }^{21,27}$, and for detunings greater than $5 \mathrm{meV}$, they observe no cavity-mode emission at low temperatures; this is expected for a single exciton-cavity coupling 28 , since the cavity-mode emission diminishes as a function of detuning.

The single QD-cavity system is realized by nucleating one InAs QD at the apex of a InP pyramid (see Fig. (4) grown using selective-area epitaxy $\stackrel{49}{\underline{4}}$. Dot formation on these InP pyramidal nanotemplates proceeds via the Stranski-Krastanow growth mode ${ }^{50}$ similar to growth on planar substrates although some subtleties of the strain distribution will differ due to the proximity of the $\{110\}$ planes that make up the sidewalls of the InP pyramid. Once planarized, however, any signature of the InP pyramid vanishes, leaving a coherent InAs dot in a uniform InP matrix. One distinction between planar and site-controlled dots pertains to the wetting layer. Although the presence of a wetting layer is as-

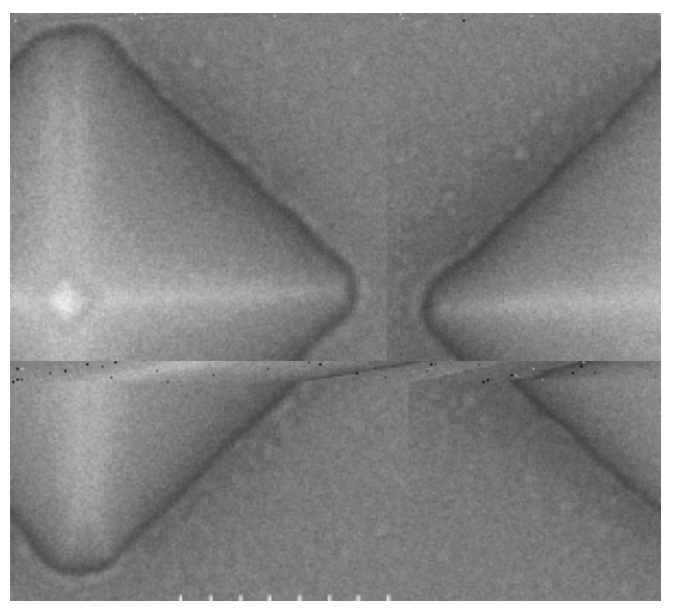

FIG. 4: A single InAs quantum dot nucleated at the apex of a InP pyramid. The pyramid is grown using selectivearea epitaxy on a electron-beam patterned $\mathrm{SiO}_{2}$-coated $\mathrm{InP}$ substrate. The scale bar is $210 \mathrm{~nm}$.
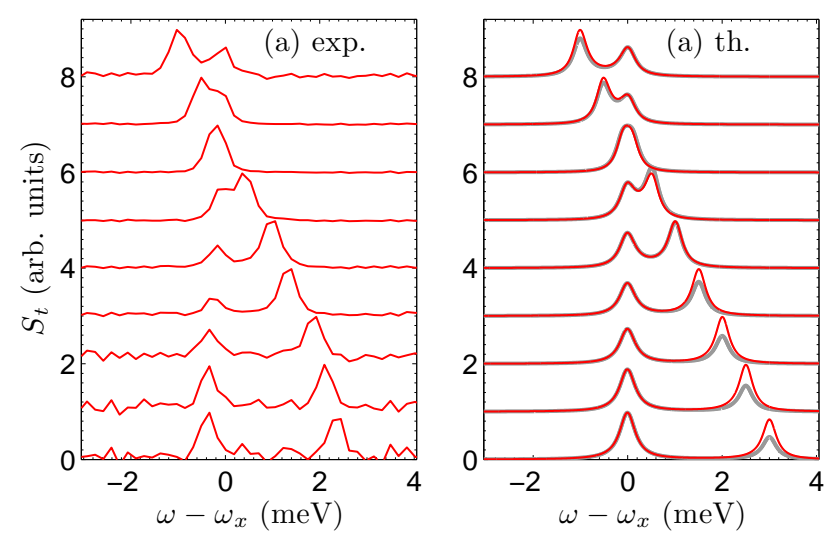

FIG. 5: (Color online) (a) Recently published experimental data (Dalacu et $a l^{29}$ ), taken at $T=4 \mathrm{~K}$, normalized to show the effect of detuning on the exciton and cavity mode. (b) Theoretical simulations with (red-dark) and without (grey-light) phonon interactions. The parameters are similar to those used in Fig. (1), except that $\Gamma^{\prime}=150 \mu \mathrm{eV}$ and we have convolved with a Lorentzian function with FWHM $\Gamma_{\text {spec }}=250 \mu \mathrm{eV}$ to account for the spectral resolution in the experiment ${ }^{29}$. The detunings are not exactly fit to experiment, but rather are chosen to cover a similar range to the experiments with equal frequency spacing. Both sets of calculations are normalized to their peak value for clarity. We also include the radiation-mode emission, with $F_{c}=2 F_{r}$.

sumed, its lateral extent is limited to the apex of the pyramid and is thus only slightly larger than the QD. The site-controlled dots are therefore expected to have a modified shape compared to planar dots, and the absence of an infinite 2D wetting layer. Although these differences to do not manifest in the electronic structure of the dots 51 , the absence of a wetting layer and associated continuum of states may have implications related to non-resonant dot-cavity coupling 27 . Clearly, reducing unknown and unwanted excitation mechanisms should be avoided from a practical perspective, which makes dots without a wetting layer advantageous, e.g., for creating cavity-assisted single photons on demand 52 .

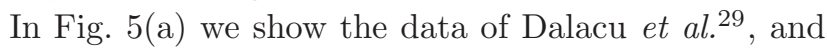
in Fig. F(b) we show our analytical model with (red-dark curve) and without (grey-light curve) phonon coupling. Evidently, the analytic phonon model well reproduces the experiment data without the need to artificially add in a cavity pump term, and the phonon coupling plays a role on the cavity feeding mechanism. This general conclusion is consistent with earlier works $15,16,18,30$, though some of the qualitative trends are quite different (e.g., a cavity suppression followed by an enhancement); moreover, since our final spectral forms are analytic, one can sweep a wide range of parameters in a straightforward and simple way. Moreover, our formulas can be applied to fit spectra over a wide range of temperatures (up to room temperature), where one must go beyond a second- 


\section{CONCLUSIONS}
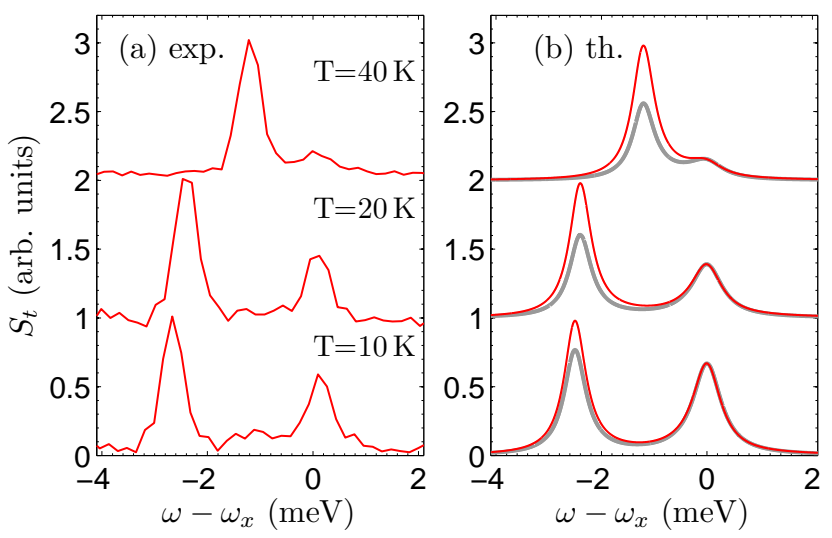

FIG. 6: (Color online) (a) Measured spectra at various temperatures, taken at $T=10 \mathrm{~K}, T=20 \mathrm{~K}$, and $T=40 \mathrm{~K}$. (b) Theoretical simulations with (red-solid curve) and without (grey-light curve) phonon interactions. The parameters are similar to those used in Fig. 5, except that $\Gamma^{\prime}=225-300 \mu \mathrm{eV}$ (increasing linearly with temperature) and we have convolved with a Lorentzian function with FWHM $\Gamma_{\text {spec }}=400 \mu \mathrm{eV}^{53}$.

order approximation for the phonon baths.

Finally, we fit the emission spectra for elevated temperatures, using data that has not previously been shown. The sample and experiment are similar to those described by Dalacu et al. 29 . In Fig. 6] we show the experimental data (a) and simulations (b) for a temperature-controller cavity detuning, for temperatures of $10 \mathrm{~K}, 20 \mathrm{~K}$, and $40 \mathrm{~K}$. Once more, we see a good trend with the experiments, and the effect of the phonons is to enhance the oscillator strength of the cavity mode relative to the exciton mode, which becomes stronger as a function of temperature.
We have presented a Green function theory to describe photon emission in arbitrary QD-cavity systems, without recourse to either the weak coupling regime or a perturbative approximation for the phonons. We have exploited this approach to model the strong coupling regime between a single exciton and a photonic crystal cavity, and, using a very simple initial condition of an inverted electron-hole pair, obtained the emission spectra for various dot-cavity detunings. Phonon related effects such as cavity-mode suppression and enhanced cavity feeding are demonstrated, showing the need to include both real and imaginary phonon self-energy terms with internal coupling. The model was then applied to help explain experimental data as a function of cavity-exciton detuning and as a function of temperature; good agreement has been found without artificially adding in a cavity-mode pump - as was done previously ${ }^{29}$. The phonon-induced cavity coupling thus mimics an incoherent cavity pump term, which naturally would be detuning dependent because of the asymmetric phonon bath. Our results and general predictions are also consistent with the recent data and polaron master equation ${ }^{11}$ simulations of Ota et al. $\stackrel{16}{ }$.

\section{Acknowledgments}

This work was supported by the National Sciences and Engineering Research Council of Canada and the Canadian Foundation for Innovation. We gratefully acknowledge U. Hohenester, Y. Ota, and C. Roy for useful discussions.
* Electronic address: shughes@physics.queensu.ca

1 A. Vagov, V. M. Axt, and T. Kuhn, Phys. Rev. B 66, 165312 (2002).

2 J. Förstner, C. Weber, J. Danckwerts, and A. Knorr, Phys. Rev. Lett. 91, 127401 (2003).

3 G. Mahan, Many-Particle Physics Plenum, New York, 1981.

4 B. Krummheuer, V. M. Axt, and T. Kuhn, Phys. Rev. B 65, 195313 (2002).

${ }^{5}$ P. Borri, W. Langbein, S. Schneider, U. Woggon, R. L. Sellin, D. Ouyang, and D. Bimberg, Phys. Rev. Lett. 87, 157401 (2001).

6 P. Machnikowski, Phys. Rev. Lett. 96, 140405 (2006).

7 E. A. Muljarov and R. Zimmermann, Phys. Rev. Lett. 93, 237401 (2004).

${ }^{8}$ G. Ortner, D. R. Yakovlev, M. Bayer, S. Rudin, T. L. Reinecke, S. Fafard, Z. Wasilewski, and A. Forchel, Phys. Rev. B 70, 201301 (R) (2004).
9 S. Rudin, T. L. Reinecke, and M. Bayer, Phys. Rev. B 74, 161305 (R) (2006).

${ }^{10}$ G. Lindwall, A. Wacker, C. Weber, and A. Knorr, Phys. Rev. Lett. 99, 087401 (2007).

11 I. Wilson-Rae and A. A. Imamoglŭ, Phys. Rev. B 65, 235311 (2002).

12 D. P. S. McCutcheon and A. Nazir arXiv:1009.1155 (2010); for related experiments, see A. J. Ramsay, T. M. Godden, S. J. Boyle, E. M. Gauger, A. Nazir, B. W. Lovett, A. M. Fox, and M. S. Skolnick, Phys. Rev. Lett. 105, 177402 (2010); A. J. Ramsay, Achanta Venu Gopal, E. M. Gauger, A. Nazir, B. W. Lovett, A. M. Fox, and M. S. Skolnick, Phys. Rev. Lett. 104, 017402 (2010).

13 F. Milde, A. Knorr, and S. Hughes, Phys. Rev. B 78, 035330 (2008).

14 J. Xue, K-D Zhu and H. Zheng, J. Phys. C 20, 3252009 (2008).

15 P. Kaer, T. R. Nielsen, P. Lodahl, A.-P. Jauho, and J. 
Mørk, Phys. Rev. Lett. 104, 157401 (2010).

16 Y. Ota, S. Iwamoto, N. Kumagai, and Y. Arakawa, arXiv:0908.0788v1 (2009).

17 U. Hohenester, Phys. Rev. B 81, 155303 (2010).

${ }^{18}$ U. Hohenester,A. Laucht, M. Kaniber, N. Hauke, A. Neumann, A. Mohtashami, M. Selinger, M. Bichler, and J. J. Finley, Phys. Rev. B 81, 201311 (2009).

19 K. Hennessy, A. Badolato, M. Winger, A. Atature, S. Falt, E. L. Hu, and A. A. Imamoglŭ, Nature 445, 896 (2007).

${ }^{20}$ R. Oulton, B.D. Jones, S. Lam, A.R.A. Chalcraft, D. Szymanski, D. OBrien, T.F. Krauss, D. Sanvitto, A. M. Fox, D.M. Whittaker, M. Hopkinson, and M.S. Skolnick, 15 Opt. Express, 17221 (2007).

21 M. Kaniber, A. Laucht, A. Neumann, J.M. Villas-Boas, M. Bichler, M.-C. Amann, and J. J. Finley, Phys. Rev. B 77, 161303(R) (2008).

22 J. Suffczynski, A. Dousse, K. Gauthron, A. Lemaitre, I. Sagnes, L. Lanco, J. Bloch, P. Voisin, and P. Senellart, Phys. Rev. Lett. 103, 027401 (2009).

${ }^{23}$ T. Tawara, H. Kamada, S. Hughes, H. Okamoto, M. Notomi, and T. Sogawa, Opt. Express 17, 6643 (2009).

24 A. Auffeves, B. Besga, J.-M. Gérard, and J-P Poizat, Phys. Rev. A 77, 063833 (2008).

${ }^{25}$ M. Yamaguchi, T. Asano, and S. Noda, Opt. Express 16, 18067 (2008).

26 A. Naesby, T. Suhr, P. T. Kristensen, and J. Mørk, Phys. Rev. A 78, 045802 (2008).

27 M. Winger, T. Volz, G. Tarel, S. Portolan, A. Badolato, K. J. Hennessy, E. L. Hu, A. Beveratos, J. Finley, V. Savona, and A. Imamoglŭ, Phys. Rev. Lett. 103, 207403 (2009).

28 S. Hughes and P. Yao, Opt. Express 17, 3322 (2009).

29 D. Dalacu, K. Mnaymneh, V. Sazonova, P. J. Poole, G. C. Aers, J. Lapointe, R. Cheriton, A. J. SpringThorpe, and R. L. Williams, Phys Rev B 82, 033301 (2010).

30 G. Tarel and V. Savona, Phys. Rev. B 81, 075305 (2010).

31 F. P. Laussy, E. del Valle, and C. Tejedor, Phys. Rev. Lett. 101, 083601 (2008).

32 A. Laucht, N. Hauke, J. M. Villas-Boas, F. Hofbauer, G. Böhm, M. Kaniber, and J. J. Finley, Phys. Rev. Lett. 103, 087405 (2009).

33 P. Yao, P. K. Pathak, E. Illes, S. Hughes, S. Münch, S. Reitzenstein, P. Franeck, A. Löffer, T. Heindel, S. Höfling, L. Worschech, and A. Forchel, Phys. Rev B. 81, 033309 (2010).

34 P. Yao, C. Van Vlack, A. Reza, M. Patterson, M.M. Dignam, and S. Hughes, Phys. Rev. B 80, 195106 (2009).

35 P. Yao, V.S.C. Manga Rao, S. Hughes, Laser \& Photonics Reviews 4, 499 (2010).

36 P. Yao and S. Hughes, Phys Rev. B 80, 165128 (2009).

37 P. Yao and S. Hughes, Opt. Express 17, 11505 (2009).

38 M. Wubs, L. G. Suttorp, and A. Lagendijk, Phys. Rev. A 70, 053823 (2004).

39 see, e.g., J. P. Reithmaier, G. Sek, A. Löffler, C. Hofmann, S. Kuhn, S. Reitzenstein, L. V. Keldysh, V. D. Kulakovskii, T. L. Reinecke, and A. Forchel, Nature (London) 432, 197 (2004); S. Ates, S. M. Ulrich, A. Ulhaq, S. Reitzenstein, A. Löffler, S. Höfling, A. Forchel, and P. Michler, Nat. Photonics 3, 724 (2009).

40 T. Calarco, A. Datta, P. Fedichev, E. Pazy, and P. Zoller, Phys. Rev. A 68, 012310 (2003).

${ }^{41}$ We use parameters from Ref ${ }^{14}$ : deformation potentials $D_{e}-D_{h}=6.5 \mathrm{eV}$, mass density $\rho=5.667 \mathrm{~g} \mathrm{~cm}^{-3}$, longitudinal sound velocity $c_{l}=3800 \mathrm{~m} \mathrm{~s}^{-1}$.

42 M. Bissiri , Baldassarri Hoger von Hogersthal G., Bhatti A.
S., Capizzi M., Frova A., Frigeri P. and Franchi S., Phys. Rev. B 62, 4642 (2000).

43 A. Garcya-Cristobal, A. W. E. Minnaert, V. M. Fomin, J. T. Devreese, A. Yu. Silov, J. E. M. Haverkort, J. H. Wolter, Phys. Status Solidi (b) 215, 331 (1999).

44 Note that the $\psi\left(t_{\infty}\right)$ has to be subtracted off as the IBM does not decay to zero for long times.

45 E. Illes and S. Hughes, Phys Rev B 81, 121310(R) (2010).

46 This $g$ corresponding to $V_{\text {eff }}=4 \times 10^{-20} \mathrm{~m}^{-1}, d=$ 60 Debye, $\varepsilon=12, A_{c}=0.7$ with $A_{c}$ the spatial and spectral coupling parameter to the antinode. Note that even at $4 \mathrm{~K}$, the interaction of phonons reduces this value of $g$ by approximatley $10 \%$, consistent with the simpler esimate using $\langle B(T=4 \mathrm{~K})\rangle \approx 0.9^{11}$.

47 H. J. Carmichael and D. F. Walls, J. Phys. A: Math. Nucl. Gen. 61552 (1973).

48 T. Tawara, H. Kamada, T. Tanabe, T. Sogawa, H. Okamoto, P. Yao, P. K. Pathak, and S. Hughes, Opt. Express 18, 2719 (2010).

49 P. J. Poole, D. Dalacu, J. Lefebvre, and R. L. Williams, Nanotechnology 21, 295302 (2010).

50 I. N. Stranski and L. von Krastanow, Akad. Wiss. Lit. Mainz Math.-Naturwiss K1. IIb 146, 797 (1939).

51 D. Kim, J. Lefebvre, J. Lapointe, M. E. Reimer, J. Mckee, P. J. Poole, and R. L. Williams, Phys. Stat. Sol. C 3, 3840 (2006).

52 P. K. Pathak and S. Hughes, Phys. Rev. B 82, 045308 (2010).

53 The larger spectral linewidth here is due to a different spectrometer with a lower spectral resolution; but the experimental set up was basically the same as the one described in $\operatorname{Ref}^{29}$. 